\title{
A Lei de Mitscherlich e a Análise da Variância em Experiências de Ãdubação
}

\author{
FREDERICO PIMENTEL GOMES \\ Escola Superior de Agricultura "Luiz de Queiroz", \\ Universidade de São Paulo
}

INDICE

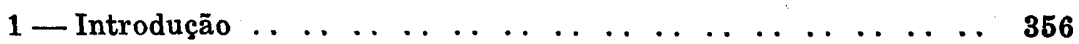

2 - A Correlação Linear e a Análise da Variância . . . . . . 356

3 - o Caso da Correlação não Linear .. . . . . . . . . . . . . . 359

4 - Uma Digressão Interessante $\ldots \ldots \ldots \ldots$

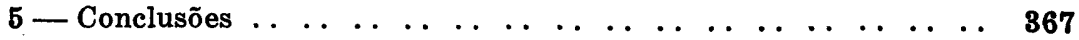

6 - Agradecimento . . . . . . . . . . . . . . . . . . . . . . . . . 368

7 - Bibliografia Citada $\ldots \ldots \ldots \ldots$ 


\section{1 - INTRODUÇÃO}

A análise da variância em certos casos é perturbada pela existência da correlação. Tal se dá, por exemplo, quando se experimentam doses diferentes de um mesmo adubo. Nêste caso, é preciso levar em conta a correlação, sem o que a análise da variância pode conduzir a resultados falsos.

Em geral se supõe que a regressão é linear. Em experiências de adubação, porém, é frequente o caso da existência de regressão não linear, geralmente do tipo exponencial introduzido por Mitscherlich. Este trabalho tem por fim especial estudar a análise da variância nesse caso.

\section{2 - A CORRELAÇÃo LINEAR E A ANÁliSE DA VARIÂNCIA}

Consideremos os seguintes dados (fictícios) onde se supõem 5 tratamentos e 4 repetições. Os valores de $x$ são as doses de adubo usadas e os $\Sigma$ y são os totais de cada tratamento.

\begin{tabular}{c|c|c|c|c|c}
\hline $\mathrm{x}$ & 0 & 1 & 2 & 3 & 4 \\
\hline$\Sigma \mathrm{y}$ & 2 & 2 & 3 & 4 & 5 \\
\hline
\end{tabular}
daria :

Uma análise da variância segundo o esquema corrente nos

\begin{tabular}{l|c|c|c}
\hline & $\begin{array}{c}\text { Soma dos } \\
\text { quadrados dos } \\
\text { desvios }\end{array}$ & $\begin{array}{c}\text { Grau de liber- } \\
\text { dade }\end{array}$ & Variância \\
\hline Tratamentos & 1,7 & 4 & 0,425 \\
Resíduo & 2,1 & 15 & 0,140 \\
\hline Total & 3,8 & 19 & \\
\hline
\end{tabular}

0 valor de $\vartheta$ será

$$
\vartheta=\sqrt{\frac{\overline{0,425}}{0,14}}=1,74 \text {, insignificante. }
$$


O fato de não levarmos em conta a correlação nos levaria a êsse resultado em desacôrdo com a realidade e equipararia os dados em apreço, onde é evidente a influência da adubação, aos seguintes, por exemplo, onde tal influência não aparece.

\begin{tabular}{r|l|l|l|l|l}
\hline $\mathrm{x}$ & 0 & 1 & 2 & 3 & 4 \\
\hline$\Sigma \mathrm{y}$ & 3 & 5 & 2 & 4 & 2 \\
\hline
\end{tabular}

Consideremos, porém, a correlação e vamos obter $r=0,65$, significativo.

A equação de regressão é

$$
y=0,2 x+0,4 \text {, }
$$

com a qual calculamos as médias esperadas.

\begin{tabular}{c|c|c|c|c|c}
\hline $\mathrm{x}$ & 0 & 1 & 2 & 3 & 4 \\
\hline $\begin{array}{c}\text { Médias es- } \\
\text { peradas }\end{array}$ & 0,40 & 0,60 & 0,80 & 1,00 & 1,20 \\
$\begin{array}{c}\text { Médias } \\
\text { observadas }\end{array}$ & 0,50 & 0,50 & 0,75 & 1,00 & 1,25 \\
\hline
\end{tabular}

A soma dos quadrados dos desvios entre as médias esperadas e as observadas dá 0,025 , número que multiplicamos por 4 ( $n^{\circ}$. de repetições). A soma dos quadrados dos desvios relativos aos tratamentos se decompõe, então, em duas partes:

\begin{tabular}{l|c|c|c}
\hline & $\begin{array}{c}\text { Soma } \\
\text { dos quadrados }\end{array}$ & $\begin{array}{c}\text { Grau de liber- } \\
\text { dade }\end{array}$ & Variância \\
\hline $\begin{array}{c}\text { Regressão } \\
\text { linear }\end{array}$ & 1,6 & 1 & 1,600 \\
$\begin{array}{c}\text { Desvios a partir } \\
\text { dos valores } \\
\text { esperados }\end{array}$ & 0,1 & 3 & 0,033 \\
\hline Tratamentos & 1,7 & 4 & 0,425 \\
\hline
\end{tabular}




\section{Temos agora}

$\eta=\sqrt{\frac{1,60}{0,14}}=3,38$, significativo.

$\mathrm{O}$ cálculo numérico poderia ser feito de maneira mais simples (1, pp. 41-43), mas a marcha seguida é mais conveniente para a compreensão do que se segue.

Como exemplo objetivo, damos a seguir a análise dos dados de uma experiência de adubação fosfatada de milho, realizada em Campinas pelo Engenheiro-Agrônomo Glauco Pinto Viegas.

\begin{tabular}{c|c|c|c|c|c}
\hline $\mathrm{x}$ & 0 & 0,25 & 0,50 & 0,75 & 1,00 \\
\hline \multirow{3}{*}{$\mathrm{y}$} & 2,82 & 3,34 & 3,90 & 4,10 & 4,68 \\
& 2,40 & 4,52 & 3,64 & 3,56 & 6,46 \\
& 2,80 & 2,80 & 3,36 & 4,30 & 4,48 \\
& 1,78 & 4,74 & 3,68 & 4,14 & 3,94 \\
\hline$\Sigma$ & 9,80 & 15,40 & 14,58 & 16,10 & 19,56 \\
\hline
\end{tabular}

Cada linha horizontal representa um bloco Os dados se referem à produção em quilos por canteiro de $30 \mathrm{~m} 2.0 x$ está expresso em quintais métricos de $\mathrm{P} 2 \mathrm{O}$ por hectare sob a forma de superfosfato. Além da adubação fosfatada, fez-se também uma adubaçao geral de $25 \mathrm{~kg} / \mathrm{ha}$ de $\mathrm{N}$ sob a forma de salitre do Chile e $45 \mathrm{~kg} / \mathrm{ha}$ de $\mathrm{K} 2 \mathrm{O}$ sob a forma de $\mathrm{KCl}$.

Temos a seguinte análise da variância, onde não isolamos a parte da variação devida aos blocos por não haver vantagem neste caso, devido à uniformidade notável dos mesmos.

\begin{tabular}{|c|c|c|c|}
\hline & $\begin{array}{c}\text { Somä dos } \\
\text { quadrados dos } \\
\text { desvios }\end{array}$ & $\underset{\text { dade }}{\text { Grau de liber- }}$ & $\begin{array}{l}\text { Quadrado } \\
\text { médio }\end{array}$ \\
\hline Tratamentos & -12, i1303 & 4 & 3,01 \\
\hline Resídup & 7,6572 & 15 & 0,51 \\
\hline Total & 19,6875 & 19 & \\
\hline
\end{tabular}


Os limites da tabela de $\vartheta$ (2, p. 523) são:

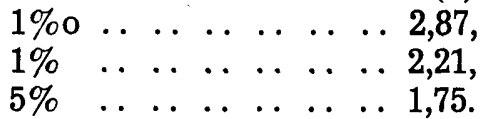
$1 \%$ o.

Logo o valor obtido é significativo para $1 \%$, mas não para

A consideração da correlação nos dá, porém, $r=0,72$, significativo.

$\mathrm{E}$ a equação de regressão é $\mathrm{y}=2,02 \mathrm{x}+2,76$.

A soma dos quadrados dos desvios devidos aos tratamentos $\mathrm{x}$ se decompõe como se segue :

\begin{tabular}{c|c|c|c}
\hline & $\begin{array}{c}\text { Soma } \\
\text { quadrados }\end{array}$ & $\begin{array}{c}\text { Grau de liber- } \\
\text { dade }\end{array}$ & $\begin{array}{c}\text { Quadrado } \\
\text { médio }\end{array}$ \\
\hline $\begin{array}{c}\text { Regressão } \\
\text { !iriear }\end{array}$ & 9,9035 & 1 &, \\
$\begin{array}{c}\text { Desvios a partir } \\
\text { dos valores } \\
\text { esperados }\end{array}$ & 2,1268 & 3 & 9,90 \\
\begin{tabular}{c} 
Tratamentos \\
\hline
\end{tabular} & 12,0303 & 4 & 0,71 \\
\hline
\end{tabular}

Temos agora

$$
\vartheta=\sqrt{\frac{9,90}{0,51}}=4,41 .
$$

Este valor de $\vartheta$ é superior ao limite de $1 \%$ o, que é 4,05 . Fica evidente, pois, a vantagem do isolamento da parcela correspondente à correlação.

\section{3 - O CASO DA CORRELAÇÃO NÃO LINEAR}

No caso de uma correlação não linear como, por exemplo, a que obedece à lei de Mitscherlich, torna-se, agora, evidente, a necessidade de uma análise da variância que leve em conta êsse fato. Utilizando os dados observados e com o auxílio das fórmulas já conhecidas (3, pp. 200-205) podemos calcular os parâmetros da função de Mitscherlich 


$$
\mathrm{y}=\mathrm{A}[1-10-\mathrm{c}(\mathrm{x}+\mathrm{b})]
$$

por meio do método dos quadrados mínimos ou do método dos momentos. Torna-se possível, então, calcular os valores esperados e, a seguir, decompor a soma dos quadrados dos desvios relativos aos tratamentos em duas partes, uma devida à regressão e outra relativa aos desvios a partir da curva de regressão. Suponhamos um caso de 5 doses diferentes de um mesmo fertilizante e 4 repetições. A soma dos quadrados dos desvios atribuidos aos tratamentos é :

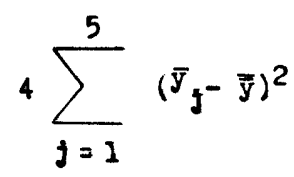

onde $\overline{\bar{y}} e^{\prime}$ a média geral, e yj é a média de cada tratamento. Seja $\hat{y}_{j} \circ$ valor esperado de acôrdo com a equação de regressão. Temos então:

$$
\begin{aligned}
& \sum_{j=1}^{5}\left(\bar{y}_{j}-y\right)^{2}=\sum_{j=1}^{5}\left(\bar{y}_{j}-\hat{y}_{j}+\hat{y}_{j}-\bar{y}\right)^{2} \\
= & \sum_{j=1}^{5}\left(\bar{y}_{j}-\hat{y}_{j}\right)^{2}+\sum_{j=1}^{5}\left(\hat{y}_{j}-\overline{\bar{y}}\right)^{2}+2 \sum_{j=1}^{5}\left(\bar{y}_{j}-\hat{y}_{j}\right)\left(\hat{y}_{j}-\bar{y}\right) \\
\text { Mas o último termo nos dá } & \left.\sum_{j=1}^{5}\left(\bar{y}_{j}-\hat{y}_{j}\right)\left(\hat{y}_{j}-\overline{\bar{y}}\right)=\sum_{j=1}^{5}\left(\bar{y}_{j}-\hat{y}_{j}\right) \hat{y}_{j}-\overline{\bar{y}} \sum_{j=1}^{5} y_{j}-y_{j}\right) \\
4 & \sum_{j=1}^{5}\left(\bar{y}_{j}-\hat{y}_{j}\right)=\sum_{i=1}^{20} y_{i}-\sum_{1}^{20} A\left[1-10-c\left(x_{I}+b\right)\right]
\end{aligned}
$$

Mas, quer no caso do método dos quadrados mínimos, quer no do método dos momentos, o segundo membro da última igualdade é nulo. Logo temos: 


$$
\begin{aligned}
4 \sum_{j=1}^{5}\left(\bar{y}_{j}-\hat{y}_{j}\right)\left(\hat{y}_{j}-\bar{y}\right) & =4 \sum_{j=1}^{5}\left(\bar{y}_{j}-\hat{y}_{j}\right) \hat{y}_{j} \\
(3,2) & =\sum_{j=1}^{20}\left\{y_{1}-A\left[1-10^{-c\left(x_{i}+b\right)}\right]\right\} \cdot\left[1-10^{-c\left(x_{j}+b\right)}\right]
\end{aligned}
$$

No caso do método dos quadrados mínimos a última expressão se anula ( 3, p. 201). Logo neste caso temos :

$$
(3,2) \sum_{j=1}^{5}\left(\bar{y}_{j}-\bar{y}\right)^{2}=\sum_{j=1}^{5}\left(\bar{y}_{j}-\hat{y}_{j}\right)^{2}+\sum_{j=1}^{5}\left(\hat{y}_{j}-\bar{y}\right)^{2}
$$

- Fica, assim, a soma dos quadrados dos desvios das médias parciais dos tratamentos repartida em duas parcelas, uma correspondente aos desvios das médias esperadas em relação à média geral, outra relativa aos desvios entre os valores observados e os valores esperados.

Quando se aplica o método dos momentos, porém, nada nos garante que a somatória de $(3,1)$ se anule. Logo a igualdade expressa em $(3,2)$ não se verifica necessàriamente e a decomposição por ela indicada é falha.

Vejamos um exemplo.

Os dados seguintes se referem a uma experiência de adubação de milho realizada em Ipanema pelos Engenheiros-Agrônomos Glauco Pinto Viegas e Erik Smith, segundo o esquema adotada para a experiência que vimos atrás.

\begin{tabular}{c|r|r|r|r|r}
\hline $\mathbf{x}$ & 0 & 0,25 & 0,50 & 0,75 & 1,00 \\
\hline & 3,38 & 7,15 & 10,07 & 9,55 & 9,14 \\
& 5,77 & 9,78 & 9,73 & 8,95 & 10,17 \\
$\mathrm{y}$ & 4,90 & $\mathbf{9 , 9 9}$ & $\mathbf{7 , 9 2}$ & 10,24 & $\mathbf{9 , 7 5}$ \\
& $\mathbf{4 , 5 4}$ & $\mathbf{1 0 , 1 0}$ & $\mathbf{9 , 4 8}$ & $\mathbf{8 , 6 6}$ & $\mathbf{9 , 5 0}$ \\
\hline$\Sigma \mathrm{y}$ & $\mathbf{1 8 , 5 9}$ & $\mathbf{3 7 , 0 2}$ & $\mathbf{3 7 , 2 0}$ & $\mathbf{3 7 , 4 0}$ & $\mathbf{3 8 , 5 6}$ \\
\hline
\end{tabular}

A análise da variância nos conduziu aos resultados seguintes, onde não se isolou a variação atribuída aos blocos por serem êstes muito uniformes, cọmo o leitor poderá verificar. 


\begin{tabular}{l|c|c|c}
\hline & $\begin{array}{c}\text { Soma dos } \\
\text { quadrados dos } \\
\text { desvios }\end{array}$ & $\begin{array}{c}\text { Grau de liber- } \\
\text { dade }\end{array}$ & $\begin{array}{c}\text { Quadrado } \\
\text { médio }\end{array}$ \\
\hline Tratamentos & 72,2199 & 4 & 18,055 \\
Resíduo & 13,6482 & 15 & 0,910 \\
\hline \multicolumn{1}{c|}{ Total } & 85,8681 & 19 & \\
\hline
\end{tabular}

$$
\vartheta=\sqrt{\frac{18,055}{0,910}}=4,45, \text { significativo. }
$$

Se procurarmos o coeficiente de correlação, acharemos $r=0,688$, significativo.

No entanto a correlação linear dá apenas uma aproximação muito grosseira, como mostram os dados e a análise que se seguem.

Correlação Linear

\begin{tabular}{c|c|c|c|c|c}
\hline $\mathbf{x}$ & 0 & 0,25 & 0,50 & 0,75 & 1,00 \\
\hline $\begin{array}{c}\text { Valores } \\
\text { esperados }\end{array}$ & 6,422 & 7,430 & 8,438 & 9,446 & 10,454 \\
$\begin{array}{c}\text { Valores } \\
\text { observados }\end{array}$ & 4,648 & 9,255 & 9,300 & 9,350 & 9,640 \\
\hline
\end{tabular}

\begin{tabular}{c|c|c|c} 
& $\begin{array}{c}\text { Soma } \\
\text { quadrados }\end{array}$ & $\begin{array}{c}\text { Grau de liber- } \\
\text { dade }\end{array}$ & Quadrado médic \\
\hline $\begin{array}{c}\text { Regressão } \\
\text { linear }\end{array}$ & 40,6499 & 1 & 40,650 \\
$\begin{array}{c}\text { Desvios a partir } \\
\text { dos valores } \\
\text { esperados }\end{array}$ & 31,5700 & 3 & 10,523 \\
\hline Tratamentos & $\mathbf{7 2 , 2 1 9 9}$ & 4 & \\
\hline
\end{tabular}




$$
\vartheta=\sqrt{\frac{\overline{40,650}}{0,910}}=6,68 \text {, significativo. }
$$

Porém os desvios a partir dos valores esperados nos dão uma nova estimativa da variância, que não deve diferir significativamente da que nos dá o resíduo. No entanto temos

$$
\vartheta=\sqrt{\frac{\overline{10,523}}{0,910}}=3,4, \text { significativo, }
$$

o que nos mostra que a correlação linear é, neste caso, pouco satisfatória. Aliás, o gráfico anexo confirma o que aí fica dito.

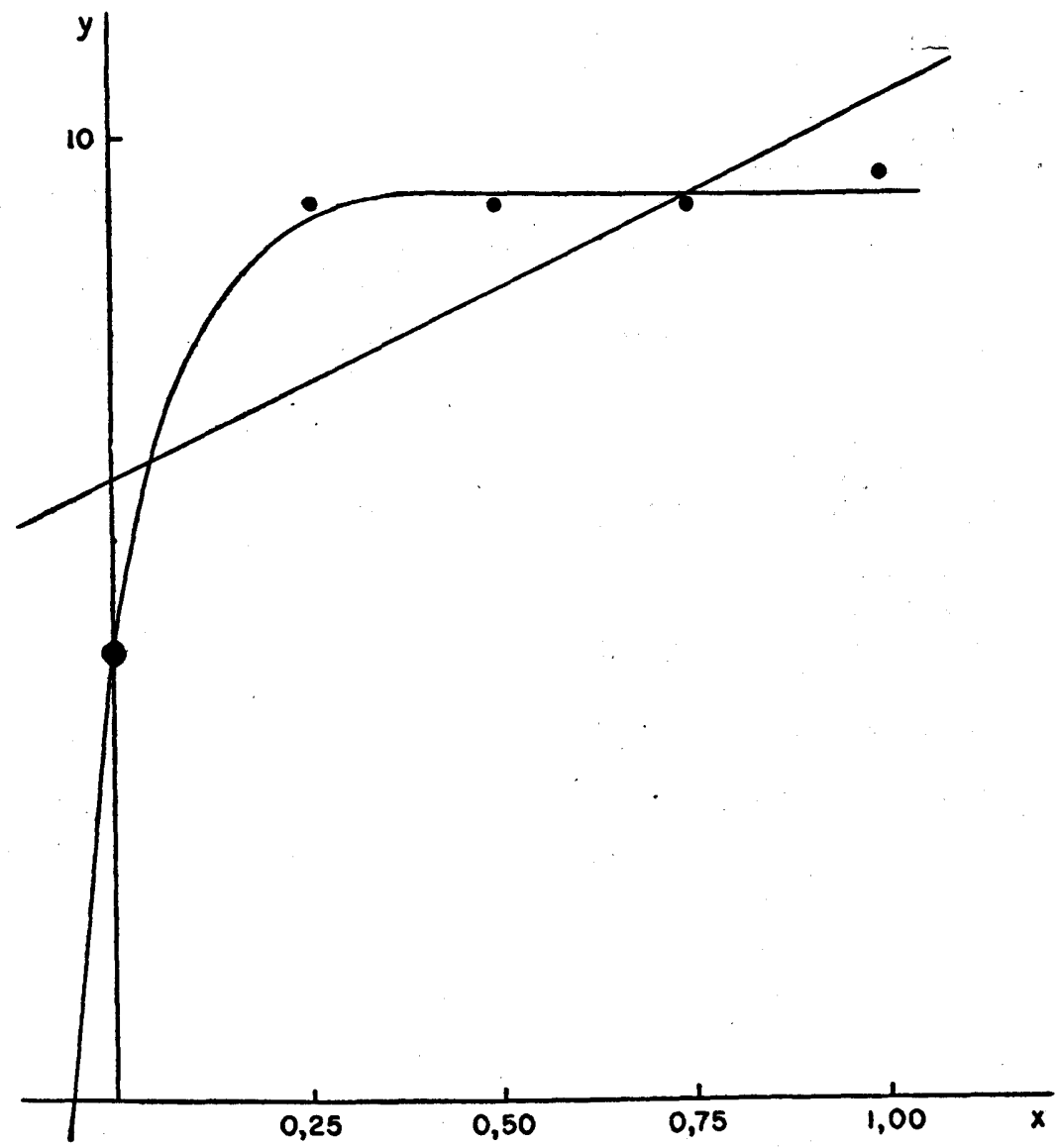

0 gráfico acima representa a reta de regressão calculada, bem como a curva de Mitscherlich interpolada pelo método dos quadrados mínimos, e ainda os valores observados, que estão indicados por pontos. 
O método dos quadrados mínimos aplicado à lei de Mitscherlich nos dá, porém, para equação de regressão

$$
y=9,436[1-10-5,590(x+0,0527)],
$$

com a qual calculamos os valores esperados que figuram no quadro seguinte

Método dos quadrados mínimos

\begin{tabular}{c|c|c|c|c|c}
\hline $\mathbf{x}$ & $\mathbf{0}$ & 0,25 & 0,50 & $\mathbf{0 , 7 5}$ & 1,00 \\
\hline $\begin{array}{c}\text { Valores } \\
\text { esperados }\end{array}$ & 4,648 & $\mathbf{9 , 2 4 4}$ & $\mathbf{9 , 4 2 8}$ & $\mathbf{9 , 4 3 6}$ & $\mathbf{9 , 4 3 6}$ \\
$\begin{array}{c}\text { Valores } \\
\text { observados }\end{array}$ & $\mathbf{4 , 6 4 7}$ & $\mathbf{9 , 2 5 5}$ & $\mathbf{9 , 3 0 0}$ & $\mathbf{9 , 3 5 0}$ & $\mathbf{9 , 6 4 0}$ \\
\hline
\end{tabular}

A análise da variância nos dá então o seguinte quadro .

\begin{tabular}{c|c|c|c}
\hline & $\begin{array}{c}\text { Soma } \\
\text { dos quadrados }\end{array}$ & $\begin{array}{c}\text { Grau de liber- } \\
\text { dade }\end{array}$ & $\begin{array}{c}\text { Quadrado } \\
\text { médio }\end{array}$ \\
\cline { 2 - 3 } $\begin{array}{c}\text { Regressão } \\
\text { pela lei de }\end{array}$ & 71,9432 & 2 & 35,978 \\
$\begin{array}{c}\text { Mitscherlich } \\
\text { Desvios a partir } \\
\text { dos valores } \\
\text { esperados }\end{array}$ & 0,2621 & 2 & 0,131 \\
\hline Tratamentos & 72,2199 & 4 & 18,055 \\
\hline
\end{tabular}

A soma $71,9432+0,2621$ difere um pouco do total dos tratamentos 72,2199 devido aos pequenos desvios inerentes às aproximações feitas.

Temos agora

$$
\begin{aligned}
& \vartheta=\sqrt{\frac{35,978}{0,910}}=6,29, \text { significativo, } \\
& \vartheta=\sqrt{\frac{0,131}{0,910}}=0,379, \text { não significativo }
\end{aligned}
$$


A análise da variância nos mostra, pois, que a correlação obedece muito bem à lei de Mitscherlich.

Se quizéssemos isolar a influência da variação entre blocos, teriamos o quadro a seguir. Como já dissemos, isso não convém neste caso, mas em geral é vantajoso.

\begin{tabular}{l|c|c|c}
\hline & $\begin{array}{c}\text { Soma } \\
\text { dos quadrados }\end{array}$ & $\begin{array}{c}\text { Grau de liber- } \\
\text { dade }\end{array}$ & $\begin{array}{c}\text { Quadrado } \\
\text { médio }\end{array}$ \\
\hline Blocos & 2,7349 & 3 & 0,912 \\
$\begin{array}{l}\text { Regressão pela } \\
\text { lei de Mitscher- } \\
\text { lich }\end{array}$ & 71,9432 & 2 & 35,978 \\
$\begin{array}{l}\text { Desvios a partir } \\
\text { dos valores } \\
\text { esperados }\end{array}$ & 0,2621 & 2 & 0,131 \\
$\begin{array}{c}\text { Resíduo } \\
\text { Total }\end{array}$ & 10,9133 & 12 & 0,909 \\
\hline
\end{tabular}

A validez de $(3,2)$ é um bom argumento a favor do método dos quadrados mínimos. Além disso a interpolação por êsse método nos dá a melhor concordância possível entre os valores esperados e os observados e, se admitirmos que seja normal a distribuição dos desvios entre êsses valores, êle é equivalente à determinação dos parâmetros pelo método da máxima verossimilhança ("maximum likelihood") $\left(4,2^{\circ}\right.$. vol., p. 59$)$. O método dos momentos, porém, conduz a uma equação muito mais simples. Por exemplo no caso em estudo o método dos quadrados mínimos nos dá uma equação do quarto grau em $\mathrm{z}=10-0,25 \mathrm{c}$, ao passo que o método dos quadrados mínimos nos leva, depois de operações muito mais laboriosas, e uma equação do décimo grau. E' possível, portanto, que muitos dêem preferência ao método dos momentos que, no exemplo em estudo, nos conduz à equação

$$
\mathrm{y}=9,553[1-10-3,470(\mathrm{x}+0,086)]
$$

Com esta equação obtivemos os valores esperados do quadro seguinte. 
Método dos momentos

\begin{tabular}{c|c|c|c|c|c}
\hline $\mathbf{x}$ & 0 & 0,25 & 0,50 & 0,75 & 1,00 \\
\hline $\begin{array}{c}\text { Valares } \\
\text { esperados }\end{array}$ & 4,748 & 8,901 & 9,465 & 9,541 & $\mathbf{9 , 5 5 2}$ \\
$\begin{array}{c}\text { Valores } \\
\text { observa- } \\
\text { dos }\end{array}$ & 4,647 & 9,255 & 9,300 & 9,350 & 9,640 \\
\hline
\end{tabular}

Para avaliar o grau de concordância da curva calculada podemos obter a soma dos quadrados dos desvios entre os valores observados e os esperados

$$
\Sigma(\overline{y j}-\hat{\mathrm{yj}})^{2} .
$$

Esta soma, multiplicada pelo número de repetições e dividida pelo grau de liberdade, nos dá uma estimativa da variância, que não deverá diferir significativamente da que se obtém do resíduo.

No exemplo em estudo temos

$$
\frac{4 \Sigma(\overline{\mathrm{yj}}-\hat{\mathrm{yj}})^{2}}{2}=0.414
$$

Logo

$\vartheta=\sqrt{\frac{0,414}{0,910}}=0,674$, não significativo.

A interpolação é, portanto, satisfatória.

\section{4 - UMA DIGRESSAO INTERESSANTE}

Em um trabalho anterior (5, pp. 7-9) apresentamos uma fórmula para o cálculo da adubação econômicamente aconselhável. Tal fórmula era 


$$
\log \frac{f t 0,4343}{s c}=\log A-c(x+b),
$$

onde $x$ é a dose de adubo a ser usada, $\mathrm{A}, \mathrm{b}, \mathrm{c}$ são os parâmetros da equação de Mitscherlich, $t$ é o preço de um quintal métrico de adubo, $s$ é o preço de igual quantidade do produto agrícola obtido, e $f$ é um fator maior que um.

A aplicação dessa fórmula aos dados em estudo, suposto o preço de 750 cruzeiros por quintal-métrico de $\mathrm{P} 2 \mathrm{O5}$ e de 100 cruzeiros por quintal de milho e ainda tomando-se $f=1,5$, nos dá

$$
\mathrm{x}=0,132
$$

no caso do método dos quadrados mínimos. Conclui-se, então, que a adubação mais aconselhável seria a de cêrca de 13 quílos de $\mathrm{P} 2 \mathrm{O} 5$ por hectare, isto é, uns 72 quilos de superfosfato com $18 \%$ de P2O5 por hectare. Esta adubação é evidentemente pobre. Isso se deve aos seguintes fatores:

I. Elévádo preço do adubo;

II. Baixo preço do milho;

III. Valor elevado de $c$.

Com efeito o valor de $c$ obtido, corresponde, se referido a quintais-métricos de milho por hectare, a 1,677, quando Mitscherlich obtinha em média, na Alemanha, 0,60 e CROWTHER $(6$, p. 96$)$ conseguia na Grã-Bretanha um valor correspondente a 0,64 . Porém SARAIVA $(7 ;$ p. 26) obteve no Rio de Janeiro um valor de $c$ que, reduzido às unidades usadas por Mitscherlich, corresponde a 1,532, valor que pouco difere do que agora indicamos.

O método dos momentos nos dá para dose mais aconselhável de adubo $\mathrm{x}=0,152$, isto é, cêrca de 15 quilos de $\mathrm{P} 2 \mathrm{O5}$ por hectare.

\section{5 - CONCLUSÕES}

A análise da variância pode ser utilizada com vantagens no estudo das experiências de adubação com o auxílio da lei de Mitscherlich. Ela nos permite avaliar a precisão da concordância entre os valores esperados e os observados e verificar se há ou não vantagem e conveniência na aplicação daquela lei. Uma vez obtida uma concordância razoável, podem-se tirar conclu- 
sões bem fundadas sôbre a adubação econômicamente mais aconselhável. Ao contrário a aplicação indiscriminada da lei de Mitscherlich, como de qualquer processo interpolatório, sem os cuidados necessários, é suspeita.

\section{6 - AGRADECIMENTO}

Agradecemos as indicações do Prof. W. L. Stevens que, além de examinar a nossa sugestão sôbre a análise da variância no caso de aplicação da lei de Mitscherlich, ainda nos deu conhecimento do importante e moderno trabalho de CROWTHER (6). Agradecemos também cordialmente o auxílio do Engenheiro-Agrônomo Izaías Rangel Nogueira, Assistente de Matemática da E. S. A. "Luiz de Queiroz", nos laboriosos cálculos realizados, e a colaboração de minha esposa no tedioso trabalho datilográfico. E finalmente, "last but not least", ficamos gratos ao Engenheiro-Agrônomo Glauco Pinto Viegas pelos dados que gentilmente nos forneceu.

\section{7 - BIBLIOGRAFIA CITADA}

1-WISHART, J. e H. G. Sanders - "Princípios e Prática de Experimentação de Campo". Tradução de G. P. Viegas. S. Paulo.

2-BRIEGER, F. G. - "Limites Unilaterais e Bilaterais na Análise Estatística”. Bragantia 6, pp. 479-545, 1946. Campinas.

3-PIMENTEL GOMES, Frederico e Eurípedes Malavolta "Aspectos Matemáticos e Estatísticos da Lei de Mitscherlich". Anais da Escola Superior de Agricultura "Luiz de Queiroz", vol. 6, (1949), pp. 193-229. Piracicaba.

4- KENDALL, Maurice G. - "The Advanced Theory of Statistics". 3a. edição, 1947. Londres.

5-PIMENTEL GOMES, Frederico e Eurípedes Malavolta "Considerações Matemáticas sôbre a Lei de Mitscherlich". 1949. Piracicaba.

6-CROWTHER, E. M. e F. Yates - "Fertilizer Policy in WarTime: The Fertilizer Requirements of Arable Crops". The Empire Journal of Experimental Agriculture, vol. IX, 1941, pp. $77-97$. Oxford.

7-SARAIVA, Mario, Admar Lopes da Cruz e Carlos del Negro - "Contribuição para o Estudo dos Methodos de Mitscherlich, Wiessmann e Neubauer". 1937. Rio de Janeiro. 